\title{
Synthesis of pyrazolopyrimidinones using a "one-pot" approach under microwave irradiation
}

\author{
Mark Kelada ${ }^{1}$, John M. D. Walsh ${ }^{1}$, Robert W. Devine ${ }^{1}$, Patrick McArdle ${ }^{2}$ \\ and John C. Stephens ${ }^{* 1}$
}

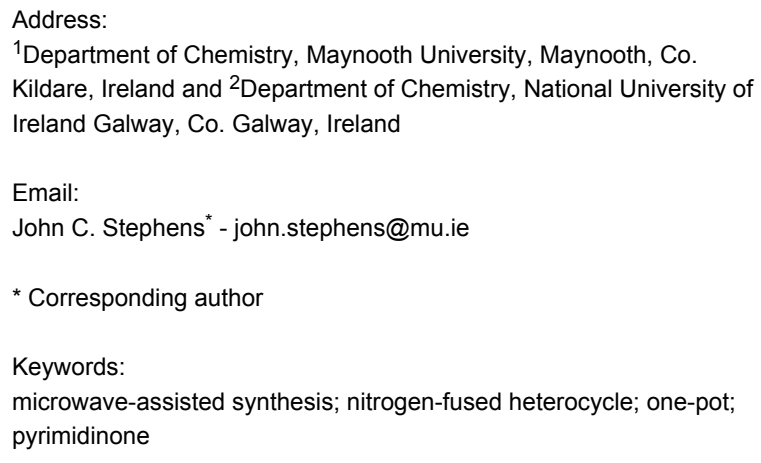

Beilstein J. Org. Chem. 2018, 14, 1222-1228. doi:10.3762/bjoc. 14.104

Received: 14 February 2018

Accepted: 04 May 2018

Published: 28 May 2018

Associate Editor: T. J. J. Müller

(c) 2018 Kelada et al.; licensee Beilstein-Institut. License and terms: see end of document.

\begin{abstract}
A simple one-pot method for the microwave-assisted synthesis of substituted pyrazolo[1,5- $a$ ]pyrimidinones, a core scaffold in many bioactive and pharmaceutically relevant compounds, has been established. A variety of substituents was tolerated at the 2 and 5 positions, including functionalized aryls, heterocycles, and alkyl groups.
\end{abstract}

\section{Introduction}

The pyrazolo[1,5- $a]$ pyrimidinone is a fused nitrogen-containing heterocyclic system and is of interest due to its role as a basic core scaffold in many bioactive and pharmaceutically relevant compounds, as well as its structural similarity to purine [1-4]. Pyrazolo[1,5-a]pyrimidinone derivatives have found use in the battle against several illnesses including cancer [5], viral infections [6-8], obesity [9], and cystic fibrosis [10] (Figure 1). It is these pharmacological properties, coupled with the pharmaceutical and fine chemical industries interest in synthetic processes that utilize cleaner and more efficient technology, which has stimulated the search for improved synthetic procedures for their generation.

Consequently, strategies for the synthesis of compounds of value, such as the pyrazolo[1,5-a]pyrimidinones, that employ one-pot syntheses and/or processes have been encouraged and have become a major focus for many synthetic chemists [11]. As such, a convenient and general method of preparing the scaffold would be of significant interest to the pharmaceutical industry. Herein, we report such a strategy for the simple synthesis of functionalized pyrazolo $[1,5-a]$ pyrimidinones that employs a one-pot microwave-assisted approach.

\section{Findings}

A number of synthetic approaches have been developed for the synthesis of privileged nitrogen-fused bicyclic systems such as the pyrazolo[3,4- $b]$ pyridines, pyrazolo[1,5-a]pyrimidines and pyrazolo[1,5-a]pyrimidinones [12-18]. The majority of methods used in the generation of the pyrazolo $[1,5-a]$ pyrimidinones employ a two-step process, which first requires the synthesis 
<smiles>Cc1nn2c(=O)cc(N3CCOC(C)C3)[nH]c2c1Cc1cccc(C(F)(F)F)c1C</smiles>

GSK WO2013028263 anticancer<smiles>C=CCc1c[nH]c2c(-c3ccc(Cl)cc3)c(-c3ccccc3Cl)nn2c1=O</smiles>

Pfizer WO2005103052 antiobesity appetite supressor<smiles>CCc1c(C)[nH]c2cc(C3CCCCN3C(=O)c3cc(F)ccc3NS(C)(=O)=O)nn2c1=O</smiles>

Gilead WO2011163518 antiviral<smiles>CCN(CCNC(=O)c1cnn2c(=O)c(Cc3ccccc3F)c(C)[nH]c12)c1ccccc1</smiles>

Philli WO2007005541 antiviral<smiles>COC(=O)Cc1c(C)[nH]c2cc(-c3cccc(Br)c3)nn2c1=O</smiles>

BMS WO2014028384

HIV intermediate

Figure 1: Bioactive pyrazolo[1,5-a]pyrimidinones.

and isolation of the intermediate 5 -aminopyrazoles followed by their reaction with an appropriate $\beta$-dicarbonyl compound [1-4,19-29]. A very recent and excellent review by Aggarwal et al. covers the use and versatility of 5-aminopyrazoles in the synthesis of a range of pyrazoloazines [30].

As part of a study into the development of novel biologically active compounds based on the pyrazolopyrimidinone scaffold, we sought to develop a simple one-pot synthesis of the nitrogen-fused bicyclic system. In order to establish reaction conditions for this one-pot synthesis, we began by seeking general reaction conditions for the microwave-assisted generation of the intermediate 5-aminopyrazoles. Once established, this methodology could be expanded to generate a one-pot synthesis of pyrazolo[1,5-a]pyrimidinones.

Typical methods for preparing 5-aminopyrazoles require conventional heating of the appropriate $\beta$-ketonitrile in the presence of a hydrazine [1-4,19-29], with Rao et al. [31] and Bagley et al. [32] reporting microwave-assisted protocols, the former requiring an acid catalyst. In order to conduct a solvent screen we chose $\beta$-ketonitrile 1a as a model substrate and reacted it with hydrazine, employing a constant temperature of $150{ }^{\circ} \mathrm{C}$ and a reaction time of $5 \mathrm{~min}$. Results from the solvent screen can be found in Table 1, with high isolated yields of the product 5-aminopyrazole 2a in all cases. As expected, a significant improvement in reaction time was observed under microwave conditions in comparison with conventional heating (Table 1 , entries 7, 8 and 9). Methanol was subsequently selected as the solvent of choice, due to the higher yield generated and its lower cost when compared with acetonitrile. Temperature and time were then varied with the best combination of temperature and time found to be $150^{\circ} \mathrm{C}$ for $5 \min$ (Table 2, entry 5).

Table 1: Solvent screen for the synthesis of 5-aminopyrazole $2 \mathbf{a}^{\mathrm{a}}{ }^{\mathrm{a}}$

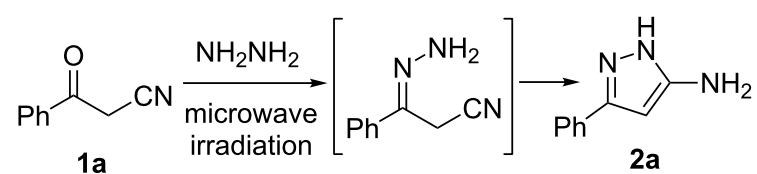

\begin{tabular}{|c|c|c|c|}
\hline Entry & Heating method & Solvent & Yield ${ }^{\mathrm{b}} \%$ \\
\hline 1 & MW & DCM & 82 \\
\hline 2 & MW & toluene & 83 \\
\hline 3 & MW & Pet ether & 88 \\
\hline 4 & MW & EtOAc & 92 \\
\hline 5 & MW & $\mathrm{MeCN}$ & 96 \\
\hline 6 & MW & $\begin{array}{l}\text { petroleum } \\
\text { ether/MeOH 9:1 }\end{array}$ & 99 \\
\hline 7 & MW & $\mathrm{MeOH}$ & 99 \\
\hline $8^{c}$ & convent. & $\mathrm{MeOH}$ & 60 \\
\hline $9^{d}$ & convent. & $\mathrm{MeOH}$ & 30 \\
\hline
\end{tabular}

aReaction conditions: ketonitrile $(0.9 \mathrm{mmol}, 1.0$ equiv), hydrazine (1.2 mmol, 1.3 equiv), solvent $(1 \mathrm{~mL})$ were heated under microwave conditions $\left(100 \mathrm{~W}, 150^{\circ} \mathrm{C}\right)$ for $5 \mathrm{~min}$. ${ }^{\mathrm{b}}$ Isolated yield. ${ }^{\mathrm{C}}$ Reaction conditions: ketonitrile $(0.9 \mathrm{mmol}, 1.0$ equiv), hydrazine $(1.2 \mathrm{mmol}$,

1.3 equiv), solvent $(1 \mathrm{~mL})$ were heated under conventional heating conditions at reflux for $17 \mathrm{~h}$. ${ }^{\mathrm{d}}$ Reaction conditions: ketonitrile

( $0.9 \mathrm{mmol}, 1.0$ equiv), hydrazine $(1.2 \mathrm{mmol}, 1.3$ equiv), solvent $(1 \mathrm{~mL})$ were heated under conventional heating conditions at reflux for $5 \mathrm{~min}$. 
Table 2: Temperature and time variations for the synthesis of 5-aminopyrazole $2 a^{a}$

\begin{tabular}{llll} 
Entry & $T\left({ }^{\circ} \mathrm{C}\right)$ & Time $(\min )$ & Yield $^{\mathrm{b}} \%$ \\
\hline 1 & 120 & 40 & 84 \\
2 & 130 & 30 & 96 \\
3 & 130 & 5 & 53 \\
4 & 140 & 20 & 98 \\
5 & 150 & 5 & 99 \\
\hline
\end{tabular}

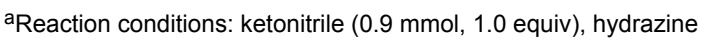
(1.2 mmol, 1.3 equiv), $\mathrm{MeOH}(1 \mathrm{~mL}$ ) were heated under microwave conditions $(100 \mathrm{~W})$. ' ${ }^{\text {Is }}$ olated yield.

A substrate scope study showed that a variety of substituents is tolerated. Aromatic groups with electron-withdrawing and electron-donating substituents at the ortho, meta and para positions were successfully explored (Scheme 1). The heterocyclic furan and thiophene substituents allowed generation of the desired 5 -aminopyrazoles in $75 \%$ and $81 \%$ yields, respectively (Scheme 1). Alkyl groups appeared to be less well tolerated, where the corresponding alkyl-substituted 5-aminopyrazoles were isolated in lower yields.

With a convenient and general microwave-assisted method for the synthesis of the 5-aminopyrazoles in hand, we next focused our attention on its application to the one-pot synthesis of pyrazolo[1,5-a]pyrimidinones.

The synthesis of pyrazolo[1,5-a]pyrimidinone 3a was chosen as the model reaction (Scheme 2). Initial reaction conditions were chosen to match the already developed microwave-assisted synthesis of the 5-aminopyrazoles. A solution of the $\beta$-ketonitrile in<smiles>Nc1cc(-c2ccccc2)n[nH]1</smiles>

$2 a$<smiles>COc1cccc(-c2cc(N)[nH]n2)c1</smiles>

$74 \%$<smiles>Cc1ccc(-c2cc(N)[nH]n2)cc1</smiles>

$70 \%$<smiles>Cc1cc(N)[nH]n1</smiles>

$2 \mathrm{~m}$

$50 \%$<smiles>Nc1cc(-c2cccc(Cl)c2)n[nH]1</smiles>

$94 \%$<smiles>COc1ccc(-c2cc(N)[nH]n2)cc1</smiles><smiles>Nc1cc(-c2ccc(Br)cc2)n[nH]1</smiles><smiles>CCc1cc(N)[nH]n1</smiles>

2n

$49 \%$<smiles>Nc1cc(-c2ccc(Cl)cc2)n[nH]1</smiles>

2c<smiles>[R]c1cc(N)[nH]n1</smiles>

2 


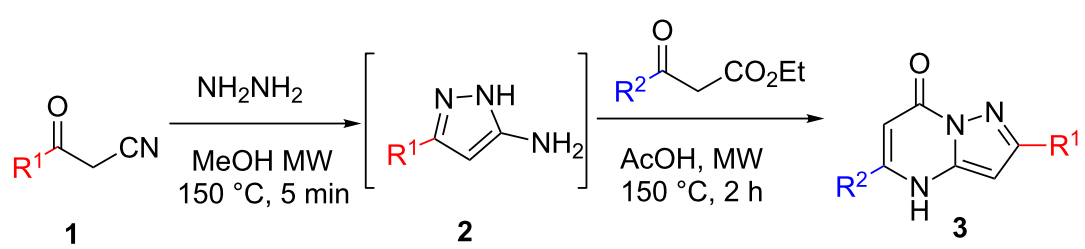<smiles>O=c1cc(-c2ccccc2)[nH]c2cc(-c3ccccc3)nn12</smiles>

$52 \%^{a, b}, 25 \%^{b, c}, 11 \%^{b, d}$<smiles>Cc1cc(=O)n2nc(-c3ccccc3)cc2[nH]1</smiles>

$3 b$

$55 \%$ a,b<smiles>O=c1cc(-c2ccc([N+](=O)[O-])cc2)[nH]c2cc(-c3ccccc3)nn12</smiles><smiles>COc1ccc(-c2cc(=O)n3nc(-c4ccccc4)cc3[nH]2)cc1</smiles><smiles>O=c1cc(-c2cc(F)c(F)c(F)c2F)[nH]c2cc(-c3ccccc3)nn12</smiles><smiles>O=c1cc(-c2ccccc2)[nH]c2cc(-c3cccc(Cl)c3)nn12</smiles><smiles>O=c1cc(-c2ccccc2)[nH]c2cc(-c3ccc(F)cc3)nn12</smiles>

$67 \%$ a,b<smiles>Cc1ccc(-c2cc3[nH]c(-c4ccccc4)cc(=O)n3n2)cc1</smiles>

$45 \%^{a, b}$<smiles>COc1ccccc1-c1cc2[nH]c(-c3ccccc3)cc(=O)n2n1</smiles>

$3 \mathrm{i}$

$32 \%^{a, b}$<smiles>CC(C)(C)c1cc2[nH]c(-c3ccccc3)cc(=O)n2n1</smiles>

$17 \%$ a,b<smiles>Cc1cc2[nH]c(-c3ccccc3)cc(=O)n2n1</smiles>

$33 \%$ a,b<smiles>O=c1cc(-c2ccccc2)[nH]c2cc(-c3cccs3)nn12</smiles>

$40 \%{ }^{a, b}$<smiles>O=c1cc(-c2ccccc2)[nH]c2cc(-c3ccco3)nn12</smiles>

$50 \%$ a,b<smiles>O=c1cc(-c2cc(C(F)(F)F)cc(C(F)(F)F)c2)[nH]c2cc(-c3ccc([N+](=O)[O-])cc3)nn12</smiles>

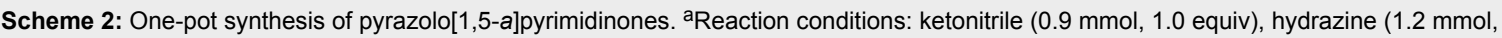
1.3 equiv), $\mathrm{MeOH}(1 \mathrm{~mL})$ were heated under microwave conditions $\left(100 \mathrm{~W}, 150^{\circ} \mathrm{C}\right)$ for 5 min followed by the addition of $\mathrm{AcOH}(0.5 \mathrm{mmol}, 0.6$ equiv) and ketoester $\left(0.9 \mathrm{mmol}, 1.0\right.$ equiv) and heated under microwave conditions $\left(100 \mathrm{~W}, 150{ }^{\circ} \mathrm{C}\right)$ for a further $2 \mathrm{~h}$. ${ }^{\mathrm{b}}$ Isolated yield. ${ }^{\mathrm{C}} \mathrm{Reactions}$ conditions: isolated aminopyrazole ( $0.9 \mathrm{mmol}, 1.0$ equiv), $\mathrm{AcOH}(0.5 \mathrm{mmol}, 0.6$ equiv), ketoester $(0.9 \mathrm{mmol}, 1.0$ equiv) in methanol ( $2 \mathrm{~mL})$ were heated using conventional heating conditions at reflux for $18 \mathrm{~h}$. ${ }^{\mathrm{d}}$ Reactions conditions: isolated aminopyrazole $(0.9 \mathrm{mmol}, 1.0 \mathrm{equiv}), \mathrm{AcOH}(0.5 \mathrm{mmol}, 0.6 \mathrm{equiv})$, ketoester $(0.9 \mathrm{mmol}, 1.0$ equiv) in methanol $(2 \mathrm{~mL})$ were heated using conventional heating conditions at reflux for $2 \mathrm{~h}$.

methanol was treated with hydrazine and heated to $150{ }^{\circ} \mathrm{C}$ under microwave irradiation for $5 \mathrm{~min}$. The $\beta$-ketoester and acetic acid were then simply added to the pot and the reaction heated at the same temperature, $150{ }^{\circ} \mathrm{C}$, under microwave irra- diation for a further $2 \mathrm{~h}$. The target pyrazolo[1,5-a]pyrimidinone was subsequently isolated in an overall yield of $52 \%$. Once more, a significant improvement was observed using microwave conditions in comparison with conventional heating. 
When a mixture of previously isolated 5-aminopyrazole 2a, $\beta$-ketoester, and acetic acid were heated under conventional refluxing conditions for $18 \mathrm{~h}$, the product pyrazolo[1,5-a]pyrimidinone 3 a could only be isolated in a $25 \%$ yield. As expected, heating the reaction mixture for $2 \mathrm{~h}$ at reflux gave a lower isolated yield of $11 \%$. A one-pot procedure under conventional refluxing conditions was also carried out in direct comparison with the microwave method, i.e., a solution of the $\beta$-ketonitrile in methanol was treated with hydrazine and refluxed for $5 \mathrm{~min}$. The $\beta$-ketoester and acetic acid were then added to the pot and the reaction refluxed for a further $2 \mathrm{~h}$. A complex mixture resulted consisting mostly of starting $\beta$-ketonitrile.

The superior performance of the microwave reaction, in terms of yield and reaction time, could result from the higher temperature and pressure achieved. The substrate scope for the one-pot reaction was then explored, with variations of both $\mathrm{R}^{1}$ and $\mathrm{R}^{2}$ groups at the pyrazolo[1,5-a]pyrimidinone core (Scheme 2 ).

Pyrazolo[1,5- $a]$ pyrimidinones containing aromatic groups with electron-withdrawing and electron-donating substituents at the ortho, meta and para positions were generated, including the multi-substituted 3n, as were those with heterocyclic furyl and thienyl substituents (Scheme 2). Alkyl-substituted pyrazolo[1,5a]pyrimidinones were also synthesized using this one-pot method (Scheme 2).

The structures of the pyrazolo[1,5- $a$ ]pyrimidinones were characterized using ${ }^{1} \mathrm{H}$ and ${ }^{13} \mathrm{C}$ NMR spectral data, HRMS, and IR spectroscopy. In addition, an X-ray crystal structure was obtained for pyrazolo[1,5-a]pyrimidinone $\mathbf{3} \mathbf{m}$ and is shown in Figure 2.

\section{Conclusion}

In conclusion, we have developed a new facile method for the one-pot microwave-assisted synthesis of substituted pyra- zolo[1,5-a]pyrimidinones, a core scaffold in many bioactive and pharmaceutically relevant compounds. A variety of functional groups was tolerated at the 2 and 5 positions, including functionalized aryls, heterocycles, and alkyl groups. Furthermore, an efficient and general microwave-assisted synthesis of versatile 5 -aminopyrazoles is reported.

\section{Supporting Information}

CCDC-1588686 contains the crystallographic data for $\mathbf{3 m}$.

The data can be obtained from The Cambridge

Crystallographic Data Centre (CCDC) via

http://www.ccdc.cam.ac.uk/data_request/cif.

\section{Supporting Information File 1}

Experimental section, NMR spectra of all synthesized compounds and crystallographic data of compound $\mathbf{3 m}$. [https://www.beilstein-journals.org/bjoc/content/ supplementary/1860-5397-14-104-S1.pdf]

\section{Acknowledgements}

We thank the Maynooth University and the Irish Research Council (IRC, IRCSET) for funding. We also thank Barbara Woods and Orla Fenelon for HRMS analysis, and Dr. Ken Maddock for his assistance with NMR analysis.

\section{ORCID ${ }^{\circledR}$ iDs}

Mark Kelada - https://orcid.org/0000-0001-9825-3417

\section{References}

1. Qi, J.; Zhang, F.; Mi, Y.; Fu, Y.; Xu, W.; Zhang, D.; Wu, Y.; Du, X.; Jia, Q.; Wang, K.; Zhang, H. Eur. J. Med. Chem. 2011, 46, 934-943. doi:10.1016/j.ejmech.2011.01.010

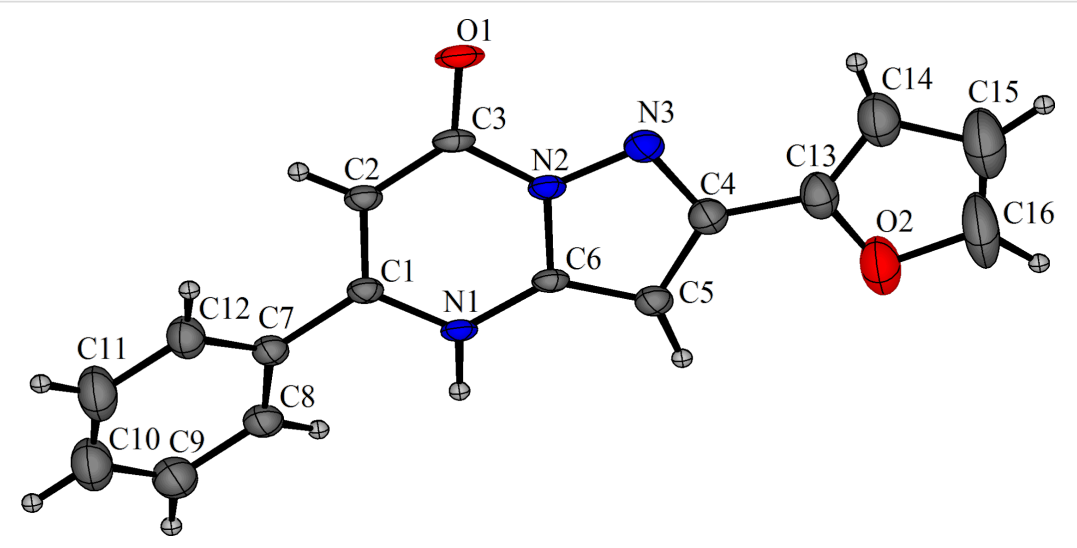

Figure 2: X-ray crystal structure of pyrazolo[1,5-a]pyrimidinone $3 \mathrm{~m}$ with ellipsoids at $50 \%$ probability. 
2. Gehling, V. S.; Bellon, S. F.; Harmange, J.-C.; LeBlanc, Y.; Poy, F.; Odate, S.; Buker, S.; Lan, F.; Arora, S.; Williamson, K. E.; Sandy, P.; Cummings, R. T.; Bailey, C. M.; Bergeron, L.; Mao, W.; Gustafson, A.; Liu, Y.; VanderPorten, E.; Audia, J. E.; Trojer, P.; Albrecht, B. K. Bioorg. Med. Chem. Lett. 2016, 26, 4350-4354. doi:10.1016/j.bmcl.2016.07.026

3. Liang, J.; Zhang, B.; Labadie, S.; Ortwine, D. F.; Vinogradova, M.; Kiefer, J. R.; Gehling, V. S.; Harmange, J.-C.; Cummings, R.; Lai, T.; Liao, J.; Zheng, X.; Liu, Y.; Gustafson, A.; Van der Porten, E.; Mao, W.; Liederer, B. M.; Deshmukh, G.; Classon, M.; Trojer, P.; Dragovich, P. S.; Murray, L. Bioorg. Med. Chem. Lett. 2016, 26 , 4036-4041. doi:10.1016/j.bmcl.2016.06.078

4. Senga, K.; Novinson, T.; Wilson, H. R.; Robins, R. K. J. Med. Chem. 1981, 24, 610-613. doi:10.1021/jm00137a023

5. Lin, H.; Moore, M. L.; Qu, J.; Rivero, R. A.; Tedesco, R.; Yu, H.; Luengo, J. I. Pyrazolopyrimidine derivative as PI3 Kinase inhibitors. WO Patent WO2013028263, Feb 28, 2013.

6. Naidu, B. N.; Patel, M.; D’Andrea, S.; Zheng, Z. B.; Connolly, T. P.; Langley, D. R.; Peese, K.; Wang, Z.; Walker, M. A.; Kadow, J. F. Inhibitors of human immunodeficiency virus replication. WO Patent WO2014028384, Feb 20, 2014.

7. Babaoglu, K.; Boojamra, C. G.; Eisenberg, E. J.; Hui, H. C.; Mackman, R. L.; Parrish, J. P.; Sangi, M.; Saunders, O. L.; Siegel, D.; Sperandio, D.; Yang, H. Pyrazolo[1,5-a]pyrimidines as antiviral agents. WO Patent WO2011/163518, Dec 29, 2011.

8. Gu, B.; Block, T.; Cuconati, A. Small molecule inhibitors against west nile virus replication. WO Patent WO2007005541, Jan 11, 2007.

9. Griffith, D. A. Pyrazolo[1,5-a]pyrimidin-7-one compounds and uses thereof. WO Patent WO2005103052, Nov 3, 2005.

10. Binch, H.; Grootenhuis, P. D. J.; Pierce, A.; Fanning, L. T. D. Modulators of cystic fibrosis transmembrane conductance regulator. WO Patent WO2009076593, June 18, 2009.

11. Volla, C. M. R.; Atodiresei, I.; Rueping, M. Chem. Rev. 2014, 114, 2390-2431. doi:10.1021/cr400215u and references cited therein.

12. El-borai, M. A.; Rizk, H. F.; Abd-Aal, M. F.; El-Deeb, I. Y. Eur. J. Med. Chem. 2012, 48, 92-96. doi:10.1016/j.ejmech.2011.11.038

13. Quiroga, J.; Portilla, J.; Abonía, R.; Insuasty, B.; Nogueras, M.; Cobo, J. Tetrahedron Lett. 2008, 49, 6254-6256. doi:10.1016/j.tetlet.2008.08.044

14. Quiroga, J.; Portilla, J.; Abonía, R.; Insuasty, B.; Nogueras, M.; Cobo, J. Tetrahedron Lett. 2007, 48, 6352-6355. doi:10.1016/j.tetlet.2007.07.041

15. Hédou, D.; Deau, E.; Dubouilh-Benard, C.; Sanselme, M.; Martinet, A.; Chosson, E.; Levacher, V.; Besson, T. Eur. J. Org. Chem. 2013, 7533-7545. doi:10.1002/ejoc.201301014

16. Loidreau, Y.; Dubouilh-Benard, C.; Marchand, P.; Nourrisson, M.-R.; Duflos, M.; Buquet, C.; Corbière, C.; Besson, T. J. Heterocycl. Chem. 2013, 50, 1187-1197. doi:10.1002/jhet.1716

17. Rimland, J.; Dunne, A.; Hunjan, S. S.; Sasse, R.; Uings, I.; Montanari, D.; Caivano, M.; Shah, P.; Standing, D.; Gray, D.; Brown, D.; Cairns, W.; Trump, R.; Smith, P. W.; Bertheleme, N.; D'Alessandro, P.; Gul, S.; Vimal, M.; Smith, D. N.; Watson, S. P. Bioorg. Med. Chem. Lett. 2010, 20, 2340-2343. doi:10.1016/j.bmcl.2010.01.133

18. Besson, T.; Brain, C. T. Heterocyclic Chemistry Using Microwave-Assisted Approaches. In Microwave Assisted Organic Synthesis; Tierney, J. P.; Lidström, P., Eds.; Blackwell Publishing Ltd.: Oxford, UK, 2005. doi:10.1002/9781444305548.ch3
19. Wustrow, D. J.; Capiris, T.; Rubin, R.; Knobelsdorf, J. A.; Akunne, H.; Duff Davis, M.; MacKenzie, R.; Pugsley, T. A.; Zoski, K. T.; Heffner, T. G.; Wise, L. D. Bioorg. Med. Chem. Lett. 1998, 8 , 2067-2070. doi:10.1016/S0960-894X(98)00372-2

20. Nam, N. L.; Grandberg, I. I.; Sorokin, V. I. Chem. Heterocycl. Compd. 2003, 39, 1210-1212. doi:10.1023/B:COHC.0000008268.94940.5c

21. Gavrin, L. K.; Lee, A.; Provencher, B. A.; Massefski, W. W.; Huhn, S. D.; Ciszewski, G. M.; Cole, D. C.; McKew, J. C. J. Org. Chem 2007, 72, 1043-1046. doi:10.1021/jo062120g

22. Brigance, R. P.; Meng, W.; Fura, A.; Harrity, T.; Wang, A.; Zahler, R.; Kirby, M. S.; Hamann, L. G. Bioorg. Med. Chem. Lett. 2010, 20, 4395-4398. doi:10.1016/j.bmcl.2010.06.063

23. Portilla, J.; Quiroga, J.; Nogueras, M.; Cobo, J. Tetrahedron 2012, 68, 988-994. doi:10.1016/j.tet.2011.12.001

24. Hwang, J. Y.; Windisch, M. P.; Jo, S.; Kim, K.; Kong, S.; Kim, H. C.; Kim, S.; Kim, H.; Lee, M. E.; Kim, Y.; Choi, J.; Park, D.-S.; Park, E.; Kwon, J.; Nam, J.; Ahn, S.; Cechetto, J.; Kim, J.; Liuzzi, M.; No, Z.; Lee, J. Bioorg. Med. Chem. Lett. 2012, 22, 7297-7301. doi:10.1016/j.bmcl.2012.10.123

25. Soares de Melo, C.; Feng, T.-S.; van der Westhuyzen, R.; Gessner, R. K.; Street, L. J.; Morgans, G. L.; Warner, D. F.; Moosa, A.; Naran, K.; Lawrence, N.; Boshoff, H. I. M.; Barry, C. E., III; Harris, C. J.; Gordon, R.; Chibale, K. Bioorg. Med. Chem. 2015, 23, 7240-7250. doi:10.1016/j.bmc.2015.10.021

26. Azeredo, L. F. S. P.; Coutinho, J. P.; Jabor, V. A. P.; Feliciano, P. R.; Nonato, M. C.; Kaiser, C. R.; Menezes, C. M. S.; Hammes, A. S. O.; Caffarena, E. R.; Hoelz, L. V. B.; de Souza, N. B.; Pereira, G. A. N.; Cerávolo, I. P.; Krettli, A. U.; Boechat, N. Eur. J. Med. Chem. 2017, 126, 72-83. doi:10.1016/j.ejmech.2016.09.073

27. Albrecht, B. K.; Bellon, S. F.; Gehling, V. S.; Harmange, J. C.; Leblanc, Y.; Liang, J.; Magnuson, S.; Tsui, V.; Zhang, B. Therapeutic compounds and uses thereof. U.S. Patent US20150065522, March 5, 2015.

28. Karthikeyan, C.; Lee, C.; Moore, J.; Mittal, R.; Suswam, E. A.; Abbott, K. L.; Pondugula, S. R.; Manne, U.; Narayanan, N. K.; Trivedi, P.; Tiwari, A. K. Bioorg. Med. Chem. 2015, 23, 602-611. doi:10.1016/j.bmc.2014.11.043

29. Poreba, K.; Wietrzyk, J.; Opolski, A. Acta Pol. Pharm. 2006, 63, 189-194.

30. Aggarwal, R.; Kumar, S. Beilstein J. Org. Chem. 2018, 14, 203-242. doi: $10.3762 /$ bjoc. 14.15 and references cited therein

31. Rao, B. R.; Venkateshwarlu, G.; Sunitha, P.; Kumar, K. S. Org. Chem.: Indian J. 2008, 4, 235-239.

32. Bagley, M. C.; Davis, T.; Dix, M. C.; Widdowson, C. S.; Kipling, D. Org. Biomol. Chem. 2006, 4, 4158-4164. doi:10.1039/b611493h 


\section{License and Terms}

This is an Open Access article under the terms of the Creative Commons Attribution License

(http://creativecommons.org/licenses/by/4.0), which permits unrestricted use, distribution, and reproduction in any medium, provided the original work is properly cited.

The license is subject to the Beilstein Journal of Organic Chemistry terms and conditions:

(https://www.beilstein-journals.org/bjoc)

The definitive version of this article is the electronic one which can be found at:

doi:10.3762/bjoc. 14.104 